\title{
Webgis-Based Telecommunication Resource Management Auxiliary Wiring System
}

\author{
Jin LUO ${ }^{1,2}$, XiaoFang ZU' ${ }^{1,3}$, Guo CHEN ${ }^{2}$ \\ ${ }^{1}$ Faculty of Information engineering, China University of Geosciences, Hubei Wuhan, China \\ ${ }^{2}$ ZondyCyberT \&S Co., Ltd, Hubei Wuhan, China \\ ${ }^{3}$ Faculty of Information engineering, Wuhan Sciences University, south branch, Hubei, Wuhan \\ Email: gislj@126.com
}

\begin{abstract}
This paper introduces the necessity and superiority of auxiliary wiring WEBGIS, as well as system implementation difficulties and countermeasures. Then explained the general concept of auxiliary wiring systems, data interface response, and finally introduced the system wiring switchover function, and gave an example.
\end{abstract}

Keywords: WEBGIS, ancillary wiring systems, flow chart

\section{Introduction}

According to China Telecom overall planning, the existing machine-line resources in the 97systems will gradually transfer to the existing pipeline resource management system (ie, China Telecom Network Resource Management System) for unified management of resources and processes so as to achieve the separation of resource and process. The involved machine-line resources also need the auxiliary wiring capabilities to be integrated into the existing pipeline resource management system.

As Internet technology continuous development, the demand for GIS is growing. Using Internet and Web to publish spatial data, provide spatial data browsing, query and analysis functions has been the inevitable trend of GIS development. The current WEBGIS technology with cross-platform, low development and application management costs and extensive space characteristics, will provide effective measures for auxiliary wiring of pipeline resources. This paper introduces wire resource auxiliary wiring realization measures and process based on WEBGIS.

\section{System Design Thought}

To facilitate system extensive application in near feature, system design intended to follow Microsoft, NET Development and Web Application System's three-tier architecture ----presentation layer, logic layer and data layer. To realize automatic wiring and auxiliary wiring functions by combining GIS network resource spatial and graph data. Online business SOCKET + XML data will be obtained through 97 system and interact by the interface server and WEBGIS application service.

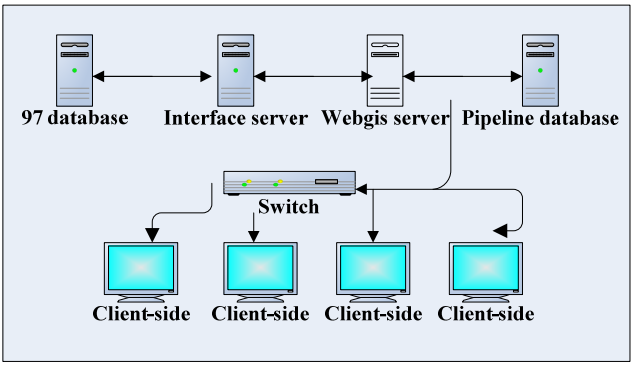

Figure 1. System physical structure chart

\section{Design Difficulties and Countermeasures}

\subsection{WEBGIS Auxiliary Wiring Design Difficulty is as Follows}

1) To achieve the basic GIS operations and analysis in the WEB page

2) Can quickly and easily access and configure the resource management system of China Telecom (Professional GIS) (C / S mode) in the resource data by the WEB; it can interact with large-scale heterogeneous 97 databases; it can realize real-time access to their online business resource occupation situation, because the wiring to be related to the occupied information modification of auxiliary wiring

3) 97 systems, professional GIS and WEBGIS has high demand in system stability, accuracy and response time, especially in 97 systems, as it is the telecommunications company's online operation system for its business and services which can't tolerate slightest error.

To solve these problems, the auxiliary wiring systems based on WEBGIS from Wuhan Zondy T\& S CO., Ltd . 
Table 1. MAPGIS_IMS6.7 list of common used components

\begin{tabular}{cl}
\hline Component name & Function description \\
\hline MpViewCom.dll & Map display, zoom in and out, Coordinate transformation, etc in WEBGIS \\
MpMapCom.dll & Realize project load, work area state, project query, etc. \\
MpAreaCom.dll & Achieve information management and query of point, line, polygon and net \\
MpAppCom.dll & Provide GIS application functions, map clip, buffer of point, line and polygon, project transfor- \\
& mation, distance and area measures, etc. \\
MpNetOperCom.dll & Provide network operation and analysis function, including network path analysis, optimize \\
MpKitCom & path, shortest path, path query, ending query, etc. \\
\hline
\end{tabular}

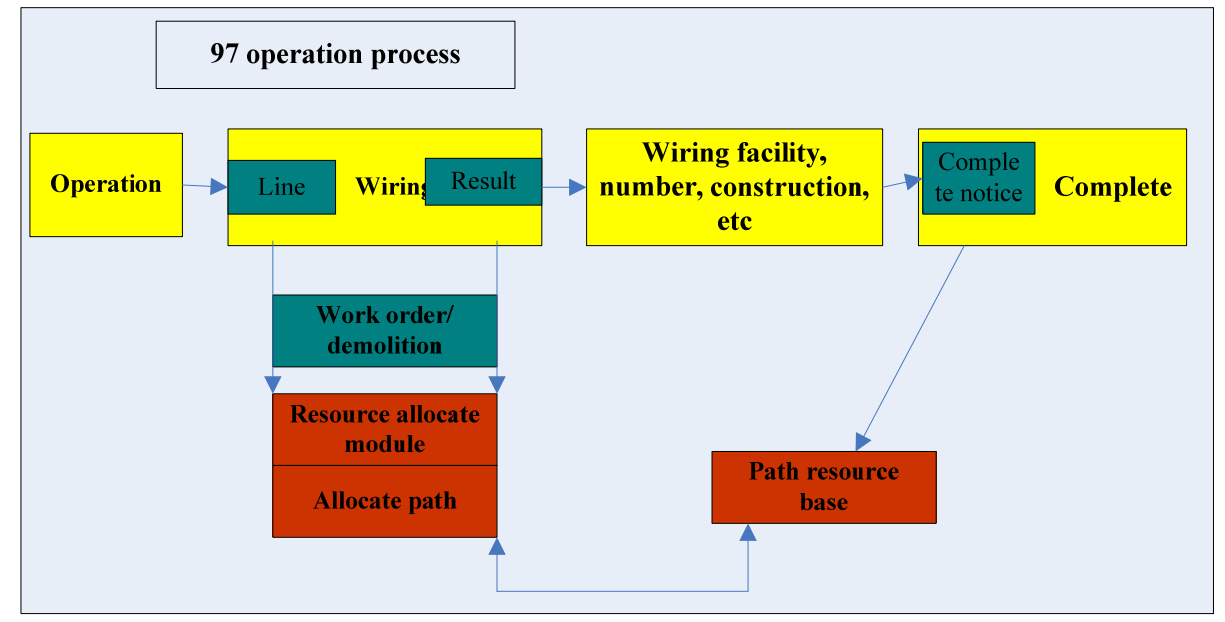

Figure 2. 97 system flow chart

new INTERENT distributed WEBGIS platform (MAPGIS-IMS) is selected.

MAPGIS-IMS based on Internet network, uses multitier architecture and integrates several distributed object technology (CORBA, DCOM, JAVA) to build and publish GIS which provides fast and integrated solutions. MPGIS-IMS provides series of COM module such as, spatial data management, map display, project management, work zone management, query and analysis to facilitate ASP.net and also to establish various GIS application solutions through $\mathrm{WEB}^{[4]}$. The common function components are shown as Table 1.

\subsection{Data Interface Countermeasures}

97 databases require timely and strong stability. To achieve heterogeneous database real-time interaction, an appropriate method is through the widely used SOCKET + XML complying with certain norms for data transmission. To run the original 97 normally without large modification, firstly, to copy all the data from resource management system from the original 97 such as, print work orders, inquiries, interfaces, etc. Use redundant data to ensure system function separately running. Finally, part of system wiring 97 functions will be replaced by WEBGIS and other resources available are from professional GIS. 97 system converts to open system and it's responsible for numbers and single-process management.

Remodel C \# component through COM component under VC and NET environment. By taking original large number of C / S functions code to save a lot of research and development time.

System technical framework diagram is as follows:

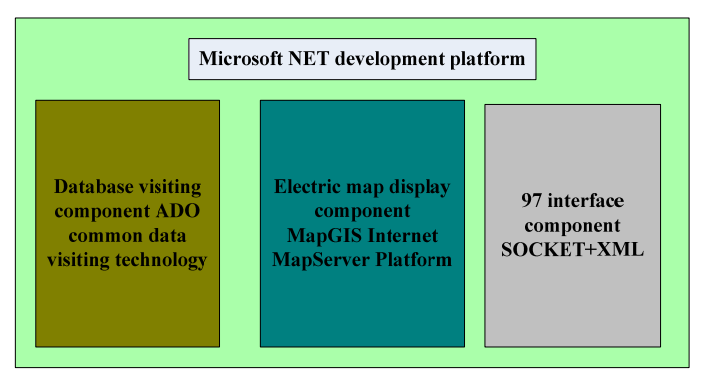

Figure 3. System technical framework diagram 


\section{System Functions ${ }^{[3]}$}

From fully research telecommunication process, we can see that the new WEBGIS auxiliary wiring is to realize wiring and cutover management.

Wiring management needs to realize the following functions, 1)Automatic with / DISCONNECT 2) artificial wiring 3) Integrated bulk wiring 4) wiring quality sampling 5) Public single fill line, user line and path query 6) wiring Log Inquiries and statistics 7) Line Completion.

These functions are not complex form technical level. Primarily, workflow needs to meet business needs.

After preliminary test, WEBGIS based wiring auxiliary can process these telecom business in WEB page, such as, newly installed or mobile telephone, an inter-bureau telephone, local telephone line, data line configuration. It supports various wiring methods, such as auto-wiring, manual wiring, alternate wiring and other wiring methods. It also support failure wiring returning and outdoor surveying, transfer and ready to install, modification, etc. It provides wiring quality sampling, fast and effective line resource configuration functions.

Cutover management needs to achieve the following main functions: 1) New cutover project 2) divide cutover range 3) cutover close up 4) cutover notification 5) cutover and the allocation of resources 6) removing the cutover 7) cutover completion.

By using GIS electric map, taking fully advantage of graphical operation, system can handle cutover operation easily, establish cutover project, divide cutover area, cutover circle, cutover resource allocation, cutover completion. These functions provide effective technical support for telecom enterprise technology innovation, municipal project, network construction and optimization.

\section{Applications}

\subsection{Manual Auxiliary Wiring}

Through the GIS system and 97interface, automatically extracting 97 systems manual wiring work orders. The system automatically matches sub-Box, wiring cables and other wiring information through the user address for user. If there is not eligible information, user can use manual intervention to match. At the same time, according to actual situation, the user can carry out operation as, to be installed, survey, focus operation, etc ${ }^{[4]}$.

(1) $\ulcorner$ Inquiries junction box close to phone number, and then match;

(2)「 According to address fuzzy matching to find sub-Box, and then matching;

(3) $\ulcorner$ Search the sub-Box within a specified radius, and then matching;

(4) $\ulcorner$ According to region to find out the respective transfer box, select the box to meet the requirements after handover, and then search the sub-Box within the jurisdiction, and then matching. After the success of wiring sent to the 97, transfer to the next link.

Wiring suspicious or failure, choose re-allocation, or transfer to be loaded links, external survey links or rewiring or re-matching.

Manual auxiliary wiring process is shown as Table 2.

\section{Conclusion}

WEBGIS technology is the GIS systems and Internet technology, combining the results. It has the following advantages:

1) thin client, a client-side burden on small.

2) No need to download software, image data of high compression ratio and speed.

3) transfer raster data, well data security.

4) client relationship between response speed and the amount of data is small, response time constant, suitable for public viewing.

5)well extension and compatibility, support WAP Search.

With the growing WEBGIS applications, GIS technology is more popular than before. Practice has proved that China Telecom's 97 aircraft WEBGIS in line reconstruction project to address the ancillary wiring

Table 2. Manual auxiliary wiring process

\begin{tabular}{cll}
\hline \multicolumn{1}{c}{ Function structure } & \multicolumn{1}{c}{ Function process } \\
\hline Extract work order & Wiring & $\begin{array}{l}\text { Receive information, batch match wiring work order } \\
\text { Receive wiring work order, access user matching transfer box and sub-box, } \\
\text { query main cables and terminal wiring cables information, arbitrarily select } \\
\text { spare state line, lastly, batch wiring and send back wiring results. } \\
\text { Extract close phone number, query matching sub-box }\end{array}$ \\
$\begin{array}{c}\text { Match } \\
\text { sub-box }\end{array}$ & Match sub-box close to phone number & $\begin{array}{l}\text { Extract user address, fuzzy matching and query sub-box according to ad- } \\
\text { dress information }\end{array}$ \\
& search the sub-Box within the jurisdiction & $\begin{array}{l}\text { Search geographic center, query sub-box according to query radius } \\
\text { Take junction box code, then query sub-box according to junction box } \\
\text { jurisdiction area }\end{array}$ \\
\end{tabular}




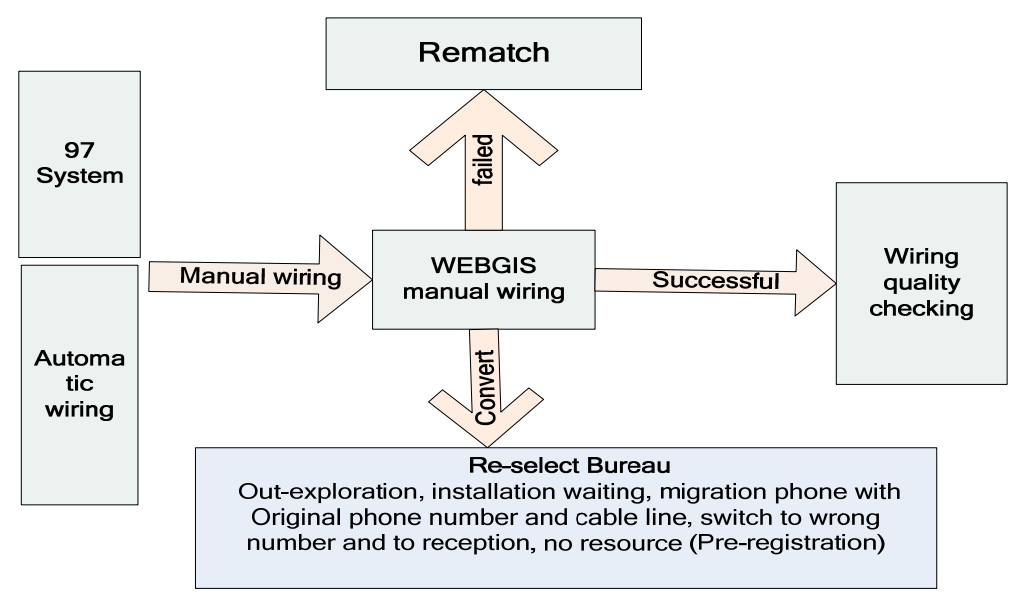

Figure 4. Functional Flow Chart

graphic which provides scientific and effective technical supports, and it provides references for telecommunications resources integration and other telecommunications operators.

\section{REFERENCES}

[1] Zhang En. The Design and reality of China Telecom Cable Resource query system based on webgis, The Master
Paper of CUG in 2005, 2005, 7.

[2] Bao Yuan Jin, Guo Jie Hua, and so on, The Research and develop of GIS based on Internet. Micro-Computer development, issue NO 2, P60-63, 1999, Feb.

[3] Chen Guo, the function design of WEBGIS Resource equipment system V1.0.doc, ZondyCyber Company Inner management document, 2005.8.23.

[4] Chen Guo, the Brief design of auxiliary configuring cable system based on WEBGIS V1.0, ZondyCyber Company Inner management document, 2005.8.12. 